(C) The Authors 2020

\title{
Addendum
}

\section{Effectiveness, quality and process evaluation of interventions to prevent childhood obesity. - ADDENDUM}

\author{
Miguel Seral-Cortes, Pilar De Miguel-Etayo, Paola Zapata and Luis Moreno-Aznar
}

doi: 10.1017/S0029665120002426, Published online by Cambridge University Press, 10 June 2020

The above mentioned article was originally published with funding information missing. This is as follows:

\section{Funding information}

This project has received funding from the European Union's Horizon 2020 research and innovation programme under the Marie Sklowdowska-Curie grant agreement $\mathrm{N}^{\circ} 801586$. This project has also received funding from the University of Zaragoza- Mecenazgo Santander.

The online version of the article has been updated to include the funding information.

\section{Reference}

Seral-Cortes, M., De Miguel-Etayo, P., Zapata, P., \& Moreno-Aznar, L. (2020). Effectiveness, quality and process evaluation of interventions to prevent childhood obesity. Proceedings of the Nutrition Society, 79(OCE2), E294. doi:10.1017/S0029665120002426 